\title{
Image ID Examined by Pathologist
}

National Cancer Institute

\section{Source}

National Cancer Institute. Image ID Examined by Pathologist. NCI Thesaurus. Code C162691.

The identifier for an image that has been examined by a pathologist. 